\title{
Nothing concentrates the mind: thoughts of death improve recall
}

\author{
Joshua Hart • Daniel J. Burns
}

Published online: 7 January 2012

(C) Psychonomic Society, Inc. 2012

\begin{abstract}
It seems likely that awareness of one's mortality is in some respects advantageous (e.g., because it helps individuals forestall death), but little research has explored the psychological mechanisms that might confer such an advantage. Recent research has shown that processing stimuli in terms of survival relevance enhances memory relative to a host of deep-processing conditions, so it is plausible that human memory has been selected to operate more efficiently when death thoughts (e.g., survival concerns) are activated. If so, then the mortality salience as a general psychological state should be sufficient to increase recall; the present experiments show this to be the case. The enhancing effect of mortality salience on recall occurred for both incidental and intentional learning tasks, relative to a variety of comparison conditions, and did not appear to be mediated by affect or arousal. Follow-up analyses revealed the effect to be mediated by the complexity of participants' elaborations about mortality. Potential theoretical implications are discussed.
\end{abstract}

Keywords Adaptive memory · Mortality salience · Survival processing $\cdot$ Free recall

Nairne, Thompson, and Pandeirada (2007) showed that orienting participants to process information in terms of its

Electronic supplementary material The online version of this article (doi:10.3758/s13423-011-0211-9) contains supplementary material, which is available to authorized users.

J. Hart $(\bowtie) \cdot$ D. J. Burns

Department of Psychology, Union College,

Schenectady, NY 12308, USA

e-mail: hartj@union.edu survival value enhances subsequent retention relative to an array of comparison conditions. Nairne et al. suggested that memory structures have been sculpted by natural selection to be specially attuned to retain information presented in survival-relevant contexts.

Nairne et al.'s (2007) findings have generated considerable interest. One characteristic of the "survival-processing" orienting task that has yet to receive much attention is that, because survival involves avoiding death, it seems likely to activate death-related cognitions. This characteristic may be important because abstract death awareness - a byproduct of human beings' comparatively sophisticated, adaptive cognitive capacities, such as self-awareness and future planning - is powerfully motivating, influencing a broad range of psychological processes (see Pyszczynski, Solomon, \& Greenberg, 2003, for a review).

Research on the effects of death awareness (i.e., as instilled by mortality salience manipulations, which encourage people to think about their own death) has demonstrated that many of these processes function to prevent or minimize the potential for death anxiety, suggesting that mortality salience is a costly encumbrance that people generally try to mitigate. Indeed, people's first response to death awareness is frequently effortful suppression (Pyszczynski, Greenberg, \& Solomon, 1999).

This tendency to regulate the extent of death concerns has potential adaptive value because chronic direct contemplation of one's unavoidable demise could be psychologically crippling and a waste of resources in the absence of direct physical danger. However, given that dying is a chief threat to any organism's chances of reproducing its genes, it seems that there should be some benefits to death-related cognitions, even if people also actively work to manage their emotional reaction to it. We suggest that there may be 
an information-processing advantage to (abstract) death awareness. ${ }^{1}$ Death is an abstract, concerning, personally relevant problem that seems likely to activate a unique cognitive context oriented toward processing information more deeply or complexly. The tendency for abstract thoughts of death to enhance the encoding and retention of information would facilitate the acquisition of skills and knowledge that could be used to directly or indirectly extend lifespan and enhance reproductive fitness in other ways.

If this is true, any items encoded subsequent to a mortality-salient state should be afforded a retention advantage. Such a finding would bear implications for a functionalist perspective on memory, and for the role of death awareness in psychological functioning more generally.

Therefore, on the basis of evidence demonstrating both the motivating role of death awareness in diverse psychological processes (e.g., Burke, Martens, \& Faucher, 2010; Hayes, Schimel, Arndt, \& Faucher, 2010) and the beneficial effects of survival processing on retention (e.g., Nairne et al., 2007), and reasoning that cognitive systems should yield some (perhaps adaptive) benefit from contemplation of lifeand-death concerns, we hypothesized that retention should be enhanced by the induction of a mortality-salient psychological state-even in the absence of explicit instructions orienting participants to the survival value of the processed stimuli.

In Experiment 1, we tested whether mortality salience would improve recall following an incidental learning task (pleasantness rating of a list of words). Experiment 2 replicated Experiment 1 using a larger, more diverse sample, as well as a comparison condition intended to control for negative affect and arousal. In Experiment 3, we tested whether mortality salience would improve retention following an intentional learning task.

\section{Experiment 1}

Method

A group of 40 undergraduate psychology students participated for credit toward a class requirement. Tested individually in small-group sessions, the participants were randomly assigned to a mortality salience (MS) condition or to a control condition that concerned watching television. Participants were asked to "Please briefly describe the emotions that the thought of your own death [watching

\footnotetext{
${ }^{1}$ To be clear, we are referring to abstract thoughts of one's own mortality, not the thoughts associated with an imminent threat to one's life. The latter would be expected to constrict information processing to concrete focal details of the immediate situation (see Christianson, 1992).
}

television] arouses in you" or to "Jot down, as specifically as you can, what you think will happen to you as you physically die [watch television] and once you are physically dead [have watched it]." This MS manipulation (Rosenblatt, Greenberg, Solomon, Pyszczynski, \& Lyon, 1989) reliably primes death-related thoughts (Hayes et al., 2010).

Participants then completed the Positive and Negative Affect Schedule (PANAS; Watson, Clark, \& Tellegen, 1988) so that we could assess whether any MS effects on recall were due to elicitation of negative or positive affect (NA or PA); previous research had suggested that affect would not play a role (e.g., Pyszczynski et al., 1999).

Next, participants rated the relative pleasantness $(1=$ extremely unpleasant, 5 = extremely pleasant) of each word in a list of 48 ostensibly unrelated words (four words were selected from each of 12 ad hoc categories). ${ }^{2}$ Embedded within the list, in Serial Positions 34 and 43, were two words relevant to death: "corpse" and "sorrow." We included these as a potential manipulation check, in case of an otherwise null effect on recall; to the extent that death thoughts remained accessible during encoding, we expected that at least these death-related words would be recalled especially well in the MS condition. ${ }^{3}$ The full list of words is presented in supplemental materials available online.

The words were shown for $6 \mathrm{~s}$ each, and participants recorded their ratings on a sheet of paper. After a delay of approximately $1 \mathrm{~min}$, during which the experimenter collected the pleasantness rating sheets and administered recall sheets, participants were given $5 \mathrm{~min}$ to freely recall as many of the previously rated words as they could.

\section{Results and discussion}

As hypothesized, the MS participants recalled more words than did participants primed with TV, $t(38)=2.51, p=.02, d=$ 0.79 (see Table 1 for the means and standard deviations). This was true for both of the death-related words, $t(38)=2.52, p=$ $.02, d=0.79$, and for the remaining 46 non-death-related words, $t(38)=2.28, p=.03, d=0.72$.

To examine whether the MS participants' recall advantage could be attributed to disproportionate recall of words rated as more or less pleasant, we computed the proportions of recall for the words rated at each point on the 1-5 pleasantness rating scale (see Table 2). For example, if a participant rated 10 words as a 1 (extremely unpleasant) and recalled 5 of those words, the participant's proportion score

\footnotetext{
${ }^{2}$ We used this list because it has been shown to produce the survivalprocessing effect (Burns, Burns, \& Hwang, 2011).

${ }^{3}$ We chose low-frequency words that were not too closely related to death so that participants would not realize that there was a connection between the MS prime and the word-rating task. The words were inserted late in the list so that they would have little effect on the encoding of the other list items, without being recency items.
} 
Table 1 Mean percentages of words recalled as a function of experimental condition across the three experiments

\begin{tabular}{llrlll}
\hline & \multicolumn{3}{l}{ Experimental Condition } \\
\cline { 2 - 3 } & \multicolumn{2}{l}{ Control Condition } & & \multicolumn{2}{l}{ Mortality Salience } \\
\cline { 2 - 3 } \cline { 5 - 6 } Experiment (Control) & M & SD & & M & \multicolumn{2}{l}{ SD } \\
\hline Experiment 1 (TV) & 39.7 & 9.08 & & 47.9 & 11.55 \\
Experiment 2 (Paralysis) & 33.3 & 14.72 & & 37.9 & 14.28 \\
Experiment 3 (Dental) & 43.7 & 15.90 & & 50.3 & 13.07 \\
\hline
\end{tabular}

for extremely unpleasant words would be .50 . A $2 \times 5$ repeated measures ANOVA showed no MS $\times$ Pleasantness interaction, $p=.13$. This remained true when we combined the negative adjectives (rated 1 or 2 ) and positive adjectives (rated 4 or 5 ), $p=.23$.

The effect of MS on recall was also not attributable to NA or PA. MS did not affect NA or PA, $p$ s $>.17$, and neither NA nor PA correlated with recall, $|r| \mathrm{s}<.19, p \mathrm{~s}>.24$. Moreover, including NA and PA as covariates in an ANOVA did not change the main effect of MS on recall $(p=.03)$.

In sum, we found that MS enhanced recall, but that the effect could not be explained by the perceived valence of items, by disproportionate retention of items rated as pleasant (or unpleasant), or by affect. However, there could have been some elevated arousal, if not affect per se, generated by MS. This is unlikely, given that prior research has found that MS did not increase physiological arousal, even as compared to neutral control conditions (Rosenblatt et al., 1989). Nevertheless, a more rigorous test of affect or arousal as mediators would include a larger sample and a more aversive and arousing comparison topic. Therefore, Experiment 2 served not only as a replication of Experiment 1, but also as a better test of arousal and affect as mechanisms mediating the effect of MS on recall, by using a control condition in which participants were asked to think about becoming physically paralyzed.

Additionally, in Experiment 2 we removed "corpse" and "sorrow" from the word list, to ensure that the effect found in Experiment 1 did not depend on death words being present in the stimulus set.

\section{Experiment 2}

\section{Method}

The participants were 166 U.S. residents recruited via the Amazon Mechanical Turk (www.mturk.com) "worker" pool and paid \$2. They ranged in age from 18 to 69 years old $(M d n=31)$. All materials and procedures were administered online using Inquisit (Millisecond Software, 2010) software, which allowed for precise control over stimulus presentation (e.g., each word from the word list) and data collection (e.g., a 4-min recall window).

The participants were randomly assigned to either the MS condition or a condition in which a parallel prompt asked them to think and write about becoming physically paralyzed (Arndt, Greenberg, Solomon, Pyszczynski, \& Schimel, 1999). Participants then completed the PANAS. Next, they rated the relative pleasantness of each word in a list of 32 ostensibly unrelated words shown for $5 \mathrm{~s}$ each. The words were a subset of the ones used in Experiment 1 (we used fewer words here because we were concerned that Internet participants would not tolerate a long study; as mentioned above, "corpse" and "sorrow" were among the words removed). Five of the participants were excluded from the analyses because they apparently did not understand the directions, failing to enter ratings for at least 31 of the 32 words.
Table 2 Mean proportions of words recalled as a function of experimental condition and pleasantness ratings (Exps. 1 and 2)

\begin{tabular}{|c|c|c|c|c|c|}
\hline & \multicolumn{5}{|c|}{ Pleasantness Ratings } \\
\hline & $\begin{array}{l}1 \\
\text { (extremely } \\
\text { unpleasant) }\end{array}$ & 2 & 3 & 4 & $\begin{array}{l}5 \\
\text { (extremely } \\
\text { pleasant) }\end{array}$ \\
\hline \multicolumn{6}{|l|}{ Experiment 1} \\
\hline $\begin{array}{l}\text { Mortality salience } \\
\text { M (SD) }\end{array}$ & $.61(.24)$ & $.53(.25)$ & $.39(.16)$ & $.46(.14)$ & $.71(.24)$ \\
\hline $\begin{array}{l}\text { Television salience } \\
\text { M (SD) }\end{array}$ & $.56(.19)$ & $.36(.24)$ & $.31(.17)$ & $.35(.18)$ & $.78(.23)$ \\
\hline \multicolumn{6}{|l|}{ Experiment 2} \\
\hline $\begin{array}{l}\text { Mortality salience } \\
\text { M (SD) }\end{array}$ & $.43(.31)$ & $.31(.29)$ & $.29(.23)$ & $.38(.23)$ & $.61(.27)$ \\
\hline $\begin{array}{l}\text { Paralysis salience } \\
\text { M (SD) }\end{array}$ & $.39(.34)$ & $.29(.31)$ & $.25(.19)$ & $.38(.22)$ & $.58(.30)$ \\
\hline
\end{tabular}


Next, participants were given a 1-min distractor task (counting Xs in arrays of random letters), and then $4 \mathrm{~min}$ to freely recall the words.

\section{Results and discussion}

As predicted, MS led to better recall than did paralysis salience, $t(159)=2.03, p=.04, d=0.32$ (see Table 1$)$, suggesting that something is mnemonically special about the mortality-salient state that cannot be easily explained in terms of simple negative affect or arousal. As in Experiment 1, affect did not explain the effect of MS on recall, as MS did not influence NA or PA, $p s>.26$; neither NA nor PA correlated with recall, $|r| \mathrm{s}<.12, p \mathrm{~s}>.15$; and the effect of MS on recall remained when affect scores were included as covariates in an ANOVA ( $p=.04)$.

As in Experiment 1, we examined recall as a function of $\mathrm{MS} \times$ Pleasantness ratings. Again, a $2 \times 5$ repeated measures ANOVA showed no MS $\times$ Pleasantness interaction, $p=.95$ (see Table 2). Furthermore, the use of the Inquisit program allowed us to assess the average response latencies to each of the processed words (i.e., the amount of time it took participants to make the pleasantness ratings). There was no difference in response latencies between the MS condition $(M=2.22 \mathrm{~s})$ and the paralysis condition $(M=2.18 \mathrm{~s}), p=.45$, indicating that the observed effect was not due to participants spending more time processing the items.

As has been the case in previous survival-processing research, we used an incidental learning task in Experiments 1 and 2 . However, to the extent that memory is generally sensitive to thoughts of death, MS should enhance recall regardless of the intention to learn. Therefore, in Experiment 3 we used an intentional learning paradigm and - despite the fact that several list-learning phenomena are attenuated with intentional learning instructions (e.g., Burns, 1996)hypothesized that MS would also enhance intentional recall.

\section{Experiment 3}

Method

A group of 91 undergraduate students participated for credit toward a course requirement. Tested individually in smallgroup sessions, the participants were randomly assigned to the MS condition or a control condition. Here, we used a different aversive and arousing comparison topic, one that is frequently used in studies priming MS: experiencing dental pain (see Burke et al., 2010). After writing about death or dental pain, participants completed the PANAS. Next, they were told that they would be shown a series of words on a projector screen, one at a time for $5 \mathrm{~s}$ each, and that they should try to remember them for a later (unspecified) memory test. We used a new word list to ensure that the effects found previously could not be attributed to the specific stimuli chosen. The list items were 36 unrelated words taken from each of 36 taxonomic categories (Van Overschelde, Rawson, \& Dunlosky, 2004). The experimenter then handed out recall sheets, read the recall instructions, and gave participants $5 \mathrm{~min}$ to freely recall the words.

Results and discussion

The participants primed with MS recalled more words than did their counterparts in the dental pain condition, $t(89)=$ 2.17, $p=.03, d=0.45$ (see Table 1). As in Experiments 1 and 2 , MS did not affect NA or PA, $p \mathrm{~s}>.33$. NA and PA were unrelated to recall, $|r| \mathrm{s}<.08, p \mathrm{~s}>.47$, and including them as covariates in an ANOVA did not eliminate the effect of MS on recall ( $p=.04)$, again suggesting that the effect of MS is not attributable to affective processes.

These results show that the MS recall advantage is not limited to incidental learning tasks, suggesting that the MS recall advantage remains, even when mnemonic processes are actively engaged during encoding.

\section{General discussion}

We found that thoughts of mortality enhanced retention of subsequently processed items relative to conditions eliciting either mundane (TV) or aversive and arousing (paralysis or dental pain) thoughts. This effect occurred for both incidental and intentional learning tasks.

The effect of MS on memory does not appear to be reductively explained by affect or arousal, both of which are generally known to influence memory in somewhat complicated ways (e.g., Deffenbacher, Platt, \& Williams, 1974; Kensinger, 2009). First, MS, at least as elicited by writing briefly about one's own death, does not generally influence self-reported affect, as seen here and in numerous previous studies (see Pyszczynski et al., 1999). Second, in Experiments 1 and 2 we demonstrated that the effect of MS does not depend on the perceived valence or pleasantness rating given to the words being processed. Third, aversive and arousing but non-death-related thoughts, such as those concerning dental pain or physical paralysis, produced inferior recall relative to $\mathrm{MS}$. Of course, we did not measure physiological arousal directly, but previous research had suggested that MS does not increase physiological arousal (Rosenblatt et al., 1989). Furthermore, research has shown that arousal tends to increase processing speed (Posner, 1978), but in Experiment 2 we found that MS did not affect response latencies. Taken together, these points suggest that a nonemotional (cognitive) component to MS is responsible for the effect. In other words, the conceptual awareness of 
death, rather than individuals' emotional response to this awareness, improves recall.

\section{Mediating mechanisms}

It is striking that merely considering the prospect of one's own mortality appears to activate a psychological state that enhances the retention of items, even when neither the items nor the kind of processing performed on those items is related to mortality (or survival). This is even more impressive when one considers that MS is not a processing manipulation, or at least not an explicit one. We think that this suggests something special about MS as a psychological state that enhances retention. But to what, precisely, could this "something special" be attributed?

We conducted follow-up exploratory analyses to try to answer this question. ${ }^{4}$ Specifically, we examined the possibility that, as compared to the control conditions we used, MS creates a context that elicits more complex, distinctive, or detailed elaborations that in turn enhance memory for subsequently processed items. For Experiments 2 and 3, three independent coders read each narrative that participants produced in response to the MS, paralysis, and dental pain prompts to rate them on a $1-5$ scale $(1=$ not at all, $5=$ extremely) for depth/meaningfulness, complexity, sophistication, detail, uniqueness, and strangeness. ${ }^{5}$ Factor and reliability analyses suggested that depth/meaningfulness, complexity, and sophistication should be clustered into a single complexity score; that uniqueness and strangeness should be clustered into a distinctiveness score; and that detail was relatively independent of the two clusters. Interrater reliability was generally good (for complexity, Chronbach's $\alpha=.77$ in Exp. 2 and .75 in Exp. 3; for distinctiveness, $\alpha \mathrm{s}=.66$ and .88 ; for detail, $\alpha \mathrm{s}=.82$ and .77).

Subsequent analyses revealed that, in Experiment 2, as compared to paralysis salience, MS increased the complexity of the narratives, $t(159)=2.32, p=.02$, as well as their distinctiveness, $t(159)=3.44, p=.001$, and had no effect on detail, $p=.57$. In Experiment 3, as compared to dental pain salience, MS again increased the complexity of the narratives, $t(89)=7.14, p<.001$, but did not affect the other variables, $p \mathrm{~s}>.53$. Additionally, mediation analyses conducted according to the guidelines of Baron and Kenny (1986) showed that in Experiments 2 and 3, narrative complexity mediated the effect of MS on recall. In both experiments, the effect of MS on recall was rendered nonsignificant $(p s>.16)$ when controlling for the coded complexity scores, while the effect of complexity scores on recall remained significant $(p s<.001)$; Sobel tests

\footnotetext{
${ }^{4}$ We thank Ken Norman for making helpful comments that led us to conduct these analyses.

${ }^{5}$ Experiment 1 had too few participants to conduct these analyses.
}

confirmed mediation in both cases: Experiment 2, $z=$ $1.96, p<.05$; Experiment 3, $z=2.72, p=.007$. Neither of the other coded variables met the critical requirements for mediation.

These results are consistent with our reasoning that the prominence of death concerns in humans creates an impetus toward information gathering. The findings are also consistent with findings suggesting that MS activates comprehension goals (Renkema, Stapel, Maringer, \& van Yperen, 2008). Perhaps abstract ruminations about one's mortality stimulate general inquisitiveness: a deep encoding set oriented toward processing information, resulting in enhanced retention. Ancestral humans for whom abstract mortality concerns caused such a tendency and consequent memory improvement would become equipped with more skills and knowledge that could be used to directly or indirectly extend lifespan or to maximize reproductive opportunities relative to humans without this tendency.

The finding that narrative complexity mediated the effect of MS on recall is intriguing, but questions remain about the precise nature of this proximal mechanism. The factor and reliability analyses referenced above suggested that no adjective used to constitute the complexity variable (depth/ meaningfulness, complexity, or sophistication) was better than the others at capturing that variable, so the construct is somewhat elusive. It is possible, for instance, that the complexity variable is simply an indicator of the extent of mortality salience itself. Similarly, we cannot be certain that narrative complexity is not a product of some other variable, such as increased motivation. Further research should examine whether the well-established motivational effects of MS (e.g., increased self-esteem striving; Pyszczynski et al., 2003) could additionally illuminate the chain of causation leading from MS to superior recall in an unrelated subsequent task.

A different possibility for future research to explore is that MS creates an elaborate (e.g., complex) encoding context whose continued activation during list presentation serves to cue retrieval of list items during later recall (e.g., Howard \& Kahana, 2002). If this is true, then the effects of death awareness might be empirically indistinguishable from other manipulations that instill elaborate encoding contexts.

\section{Concluding remarks}

Death is a constant threat and inevitable problem. Perhaps mortality reminders, in addition to being potentially burdensome, serve as a cue to individuals that intellectual complacency cannot be afforded, resulting in a motivational state in which information is sought more intently and therefore is processed more complexly and efficiently. Individuals for whom mortality salience did not cause enhanced information 
processing would not remember as much information and would not remember it as well as would individuals periodically motivated by routine reminders that life could come to an end at any moment, for any number of unforeseeable reasons.

To our knowledge, this is the first evidence pointing to a specific mechanism - memory-by which death awareness may confer benefits to humans. Presumably, the tendency for memory systems to be broadly enhanced by thoughts of mortality would be adaptive across a range of medium- and long-term situations in humans' environments of evolutionary adaptation. Perhaps this reflects a uniquely human adaptation related to the capacity for death awareness.

Additional research will be necessary to further delineate the parameters of the effect of MS on memory, to more definitively determine the underlying mechanisms responsible for the memory enhancement, and to examine whether the effect is related to the ways in which survival processing influences memory. Could it be that MS is a component of the processes that mediate the retention advantage associated with survival processing? Some studies have suggested not, showing that ancestral-environment (e.g., grasslands) survival processing produces better recall than does modernenvironment (e.g., city) survival processing (e.g., Nairne \& Pandeirada, 2010; Weinstein, Bugg, \& Roediger, 2008). However, other studies have indicated that the survivalprocessing effect may not be as unique as originally thought (e.g., Soderstrom \& McCabe, 2011). We hope that this brief report will inspire others to consider the psychological processes underpinning the intriguing effect of MS on memory.

Author note This research was supported in part by two internal faculty research grants from Union College.

\section{References}

Arndt, J., Greenberg, J., Solomon, S., Pyszczynski, T., \& Schimel, J. (1999). Creativity and terror management: Evidence that creative activity increases guilt and social projection following mortality salience. Journal of Personality and Social Psychology, 77, 19-32.

Baron, R. M., \& Kenny, D. A. (1986). The moderator-mediator variable distinction in social psychological research: Conceptual, strategic, and statistical considerations. Journal of Personality and Social Psychology, 51, 1173-1182. doi:10.1037/00223514.51.6.1173

Burke, B. L., Martens, A., \& Faucher, E. H. (2010). Two decades of terror management theory: A meta-analysis of mortality salience research. Personality and Social Psychology Review, 14, 155-195.

Burns, D. J. (1996). The bizarre imagery effect and intention to learn. Psychonomic Bulletin \& Review, 3, 254-257.
Burns, D. J., Burns, S. A., \& Hwang, A. J. (2011). Adaptive memory: Determining the proximate mechanisms responsible for the memorial advantages of survival processing. Journal of Experimental Psychology: Learning, Memory, and Cognition, 37, 206-218.

Christianson, S. A. (1992). Emotional stress and eyewitness memory: A critical review. Psychological Bulletin, 112, 284-309.

Deffenbacher, K. A., Platt, G. J., \& Williams, M. A. (1974). Differential recall as a function of socially induced arousal and retention interval. Journal of Experimental Psychology, 103, 809-811.

Hayes, J., Schimel, J., Arndt, J., \& Faucher, E. H. (2010). A theoretical and empirical review of the death-thought accessibility concept in terror management research. Psychological Bulletin, 136, 699739.

Howard, M. W., \& Kahana, M. J. (2002). A distributed representation of temporal context. Journal of Mathematical Psychology, 46, 269-299. doi:10.1006/jmps.2001.1388

Kensinger, E. A. (2009). Remembering the details: Effects of emotion. Emotion Review, 1, 99-113.

Millisecond Software. (2010). Inquisit (Version 3.0.5.0) [Computer program]. Seattle, WA: Author.

Nairne, J. S., \& Pandeirada, J. N. S. (2010). Adaptive memory: Ancestral priorities and the mnemonic value of survival processing. Cognitive Psychology, 61, 1-22. doi:10.1016/j.cogpsych.2010.01.005

Nairne, J. S., Thompson, S. R., \& Pandeirada, J. N. S. (2007). Adaptive memory: Survival processing enhances retention. Journal of Experimental Psychology: Learning, Memory, and Cognition, 33, 263-273. doi:10.1037/0278-7393.33.2.263

Posner, M. I. (1978). Chronometric explorations of mind: The third Paul M. Fitts lectures delivered at the University of Michigan, September, 1976. Hillsdale: Erlbaum.

Pyszczynski, T., Greenberg, J., \& Solomon, S. (1999). A dual-process model of defense against conscious and unconscious death-related thoughts: An extension of terror management theory. Psychological Review, 106, 835-845.

Pyszczynski, T., Solomon, S., \& Greenberg, J. (2003). In the wake of 9/ 11: The psychology of terror. Washington DC: American Psychological Association.

Renkema, L. J., Stapel, D. A., Maringer, M., \& van Yperen, N. W. (2008). Terror management and stereotyping: Why do people stereotype when mortality is salient? Personality and Social Psychology Bulletin, 34, 553-564. doi:10.1177/0146167207312465

Rosenblatt, A., Greenberg, J., Solomon, S., Pyszczynski, T., \& Lyon, D. (1989). Evidence for terror management theory: I. The effects of mortality salience on reactions to those who violate or uphold cultural values. Journal of Personality and Social Psychology, 57, 681-690.

Soderstrom, N. C., \& McCabe, D. P. (2011). Are survival processing memory advantages based on ancestral priorities? Psychonomic Bulletin \& Review, 18, 564-569. doi:10.3758/s13423-011-0060-6

Van Overschelde, J. P., Rawson, K. A., \& Dunlosky, J. (2004). Category norms: An updated and expanded version of the Battig and Montague (1969) norms. Journal of Memory and Language, 50, 289-335. doi:10.1016/j.jml.2003.10.003

Watson, D., Clark, L. A., \& Tellegen, A. (1988). Development and validation of brief measures of positive and negative affect: The PANAS scales. Journal of Personality and Social Psychology, 54, 1063-1070.

Weinstein, Y., Bugg, J. M., \& Roediger, H. L., III. (2008). Can the survival recall advantage be explained by basic memory processes? Memory \& Cognition, 36, 913-919. doi:10.3758/ MC.36.5.913 